\title{
Diagnostic and therapeutic research on ultrasound microbubble/nanobubble contrast agents (Review)
}

\author{
JING MA ${ }^{1,2^{*}}$, CHANG SONG XU ${ }^{3 *}$, FENG GAO $^{1}$, MING CHEN $^{2}$, FAN LI $^{1}$ and LIAN FANG DU ${ }^{1}$ \\ ${ }^{1}$ Department of Ultrasound, Shanghai First People's Hospital Affiliated to Shanghai Jiao Tong University \\ School of Medicine, Shanghai 200080; ${ }^{2}$ Department of Cardiovascular Ultrasound, Shanghai East Hospital \\ Affiliated to Tongji University, Shanghai 200120; ${ }^{3}$ Department of Ultrasound, \\ Huai'an First People's Hospital, Nanjing Medical University, Huai'an, Jiangsu 223300, P.R. China
}

Received May 23, 2014; Accepted December 12, 2014

DOI: $10.3892 / \mathrm{mmr} .2015 .3941$

\begin{abstract}
The contrast enhanced imaging function of ultrasound contrast agents (UCAs) has been extensively investigated using physical acoustic signatures. It has a number of novel applications, including tissue-specific molecular imaging and multi-modal imaging. In addition there are numerous other therapeutic applications of UCAs, for example as vehicles for drug or gene delivery. These uses are discussed, as well as the acoustically-induced biological effects, including ultrasound targeted microbubble destruction (UTMD). This review also explores the considerations for the safe use of UCA from an acoustic standpoint. The scope of the application of UCA has markedly expanded in recent years, and it is a rapidly growing field of medical research. The current article reviews recent advances in the diagnostic and therapeutic applications of ultrasound microbubble/nanobubble contrast agents.
\end{abstract}

\section{Contents}

1. Ultrasound imaging and therapeutic applications of UCA microbubbles as well as bioeffects

2. Diagnosis and therapeutic application of UCA nanobubbles

3. Conclusion

Correspondence to: Professor Lian Fang Du, Department of Ultrasound, Shanghai First People's Hospital Affiliated to Shanghai Jiao tong University School of Medicine, $85 \mathrm{Wu}$ Jin Road, Shanghai 200080, P.R. China

E-mail: du_lf@163.com

*Contributed equally

Key words: ultrasound contrast agent, microbubble, nanobubble, molecular imaging, therapeutic application, bioeffects
1. Ultrasound imaging and therapeutic applications of UCA microbubbles as well as bioeffects

Ultrasound (US) imaging has been an important option for diagnosis and the evaluation of the efficacy of particular therapies in a variety of diseases. It is a safe, low-cost and portable mode of imaging that provides real-time information for clinicians and researchers. The wide-spread use of US contrast agents (UCAs) has improved the resolution and sensitivity of US images. UCAs may augment the acoustic impedance $(\mathrm{Z})$ between lesions and surrounding tissues by producing highly efficient scattering of waves (1). Furthermore, when treated with high acoustic energies, UCA microbubbles (MBs) undergo non-linear oscillations leading to the emission of harmonics as a source of sound, rather than simply using passive reflection. Biological tissue does not display this degree of harmonic generation, thus the contrast generated signal is able to improve the signal-to-noise ratio, which can be exploited to produce images of superior quality (2). UCAs in current use are 2-10 $\mu \mathrm{m}$ in diameter and are stable MBs, composed of inert gases, such as perfluorocarbon, stabilized by a coating of biodegradable material, such as albumin or phospholipids (3).

Diagnostic applications of UCAs. Recently, a study into three varieties of UCAs stabilized by phospholipid that are used in clinical practice, Definity, Sonovue and MicroMarker, demonstrated that the acoustic attenuation and contrast-to-tissue ratio of these molecules are comparable at frequencies $<30 \mathrm{MHz}$ at the same concentration, although their particle size distributions, encapsulated gases and shells differ. However, at frequencies $>30 \mathrm{MHz}$, MicroMarker has higher attenuation values and contrast-to-tissue ratios than either Definity or Sonovue. Furthermore, decantation was found to be an effective method by which to alter the size distribution and concentration of native clinical microbubble populations, enabling further contrast enhancement for specific pre-clinical applications (4).

Sonovue is the most commonly used form of lipid UCA. It is approved by the food and drug administration and is nontoxic 
and harmless in humans. In addition, it produced high quality contrast imaging in a number of tissues and organs, such as the liver, kidney, thyroid, breast, heart and blood vessels (5). It is particularly valuable in the diagnosis and evaluation of tumors, the assessment of myocardial motion and the evaluation of lung consolidation. Recently, Liu et al (6) showed that the peripheral enhancement pattern of breast cancer on contrast-enhanced US may be valuable in the evaluation of peripheral and central tumor angiogenesis, and of vascular endothelial growth factor (VEGF) expression. Likewise, Sonovue contrast doppler US gave a greater definition of the margins of lesions in cervical cancer as the UCA increased the sensitivity of detection of parametrial invasion and lymph node metastases (7). US contrast enhancement imaging has also been shown to improve the detection of regional wall motion abnormalities in 2-dimensional and 3-dimensional echocardiography (8). Sartori et al (9) evaluated the diagnostic accuracy of contrast-enhanced ultrasonography in differentiating between neoplastic and non-neoplastic peripheral pleuro-pulmonary lesions, observing that 40/42 non-neoplastic lesions exhibited absence of enhancement, compared with 3/53 neoplastic lesions. Other researchers have conducted real-time US contrast imaging at varying doses of contrast agent and have demonstrated kinetic features that are indicative of invasive breast tumors, with an injection of Sonovue of either $2.4 \mathrm{ml}$ or $4.8 \mathrm{ml}(10)$.

Recently, a novel tool used to monitor Sonovue microbubble signals that also automatically measures contrast flow intensity in atherosclerotic carotid plaque neovascularization has been developed by Lisowska et al (11). The results showed that patients with preserved contrast flow through the plaque were more likely to have a history of cerebral stroke. Massive calcification of atherosclerotic plaques correlated with a history of myocardial infarction and the degree of coronary artery disease, but not with a previous cerebral stroke.

Targeted imaging and therapy with MBs. Targeted imaging of tissue-specific MBs has been explored for a number of years. These were conjugated with ligands for a variety of vascular biomarkers, including integrins that are expressed during angiogenesis. However, it has been shown that targeted ligands, which have a solid structure tethered to the $\mathrm{MB}$ surface, are able to limit the MB oscillation and reduce their harmonic signals, thus weakening US contrast imaging (12).

Therapeutic applications using MBs as vehicles for drug or gene delivery to tissues have been investigated, as well as the use of US for sonoporation, which may facilitate the entrance of drug-loaded MBs into cells. Drugs may be attached to the external surface of thin lipid monolayer bubbles by covalent or noncovalent bonds, or incorporated into liposomes that are then associated with the bubble surfaces (2).

Previous studies have also demonstrated that UCA MBs were destroyed during needle injection using a variety of syringe and needle combinations. The majority of protein-shelled MBs were destroyed above a critical pressure drop of $109 \pm 7 \mathrm{kPa}$. Lipid-shelled MBs were found to have a pressure drop threshold above which $>50 \%$ were destroyed.

The commercial lipid-shelled agent Definity was found to have a critical pressure drop of $230 \pm 10 \mathrm{kPa}$. Therefore, it may be that the use of a formula is able to preclude the unnecessary destruction of microbubble contrast agent during in vivo injections. This approach may also prevent the undesirable release of drug or gene payloads in targeted MBs therapies (13).

A higher local tissue concentration of drugs or genes may be obtained by releasing these agents from bubbles by US or US-targeted microbubble destruction (UTMD) in the immediate proximity to permeabilized cells and tissues. This approach may thus augment the effect of the drug or the efficacy of the gene transfection. Chen et al (14) demonstrated that a UTMD-based short hairpin RNA delivery system effectively induces apoptosis and inhibits proliferation of cervical cancer cells and may thus be a promising option with which to treat this disease. Li et al (15) demonstrated that the efficiency of non-augmented transfer of rAAV2 into renal carcinoma cells was low $(17.28 \pm 2.44 \%)$; however, the use of UTMD enhanced viral transfer efficiency by 2-3-fold, and enhanced viral genomic DNA $>9$ fold, without decreasing cell viability. Liu et al (16) investigated the effects of this treatment in ovarian cancer cells. They demonstrated that PTX-loaded and LHRHa-targeted MBs (TPLMBs) in combination with US $(300 \mathrm{kHz}, 0.5 \mathrm{~W} /$ $\mathrm{cm}^{2}, 30 \mathrm{sec}$ ) led to apoptosis in $41.30 \pm 3.93,67.76 \pm 2.45$ and $75.93 \pm 2.81 \%$ of cells at 24,48 and $72 \mathrm{~h}$ following this treatment, respectively. UTMD predominantly promoted the effect of targeted and PTX-loaded MB chemotherapy in ovarian cancer cells. Pu et al (17) showed that UTMD may be developed as a tool with which to facilitate the delivery of LHRH receptor-targeted and paclitaxel-loaded lipid MB chemotherapeutics in the treatment of intraperitoneal ovarian cancer xenografts. Yan et al (18) found that UTMD-mediated delivery of the Timp3 gene significantly increased Timp3 protein levels in the infarct scar and border zone of an area of myocardial ischemia at three days following the administration of UTMD compared with delivery by the non-conjugated cationic microbubble. Deng et al (19) showed that UTMD significantly increased the cytoplasmic intake of pDNA and also maintained high cell viability. The nuclear import and gene expression of phstromal-derived growth factor (phSDF)-1 $\alpha$-NFkB-transfected cells were significantly higher than those transfected with phSDF-1 $\alpha$. Compared with the $\mathrm{NF \kappa B}$-free plasmids, the quantity of $\mathrm{NF \kappa B}$ plasmids in the nucleus increased 6.5-fold and the expression of SDF-1 $\alpha$ was 4.4-fold greater. Ling et al (20) demonstrated that SDF-1 and VEGF expression in the $1.5 \mathrm{~W} / \mathrm{cm}^{2}$ and $1 \mathrm{~W} / \mathrm{cm}^{2}$ groups was significantly increased compared with the $0.5 \mathrm{~W} / \mathrm{cm}^{2}$ or the control groups (3.8 to 4.7-fold; $\mathrm{P}<0.01)$. In addition, the expression of interleukin-1 $\beta$ (IL-1 $\beta$ ) in the $1.5 \mathrm{~W} / \mathrm{cm}^{2}$ group was increased two fold compared with that in the $1.0 \mathrm{~W} / \mathrm{cm}^{2}$ group, whereas no significant change was observed in the $0.5 \mathrm{~W} / \mathrm{cm}^{2}$ group. Coronary angiography and $99 \mathrm{mTc}$-tetrofosmin scintigraphy revealed that myocardial perfusion was markedly improved following treatment with UTMD + mesenchymal stem cells (MSCs). The therapeutic effects were markedly enhanced by MSC transplantation following the myocardial microenvironmental changes induced by administration of UTMD. A study also reported that UTMD effectively transfected the human growth factor gene into target tissues and had a significant effect on the recovery of injured facial nerves (21). Yang et al (22) found that cationic liposomes were conjugated with MBs using a biotin-avidin system. Plasmids carrying the most effective artificial miRNA sequences were delivered 


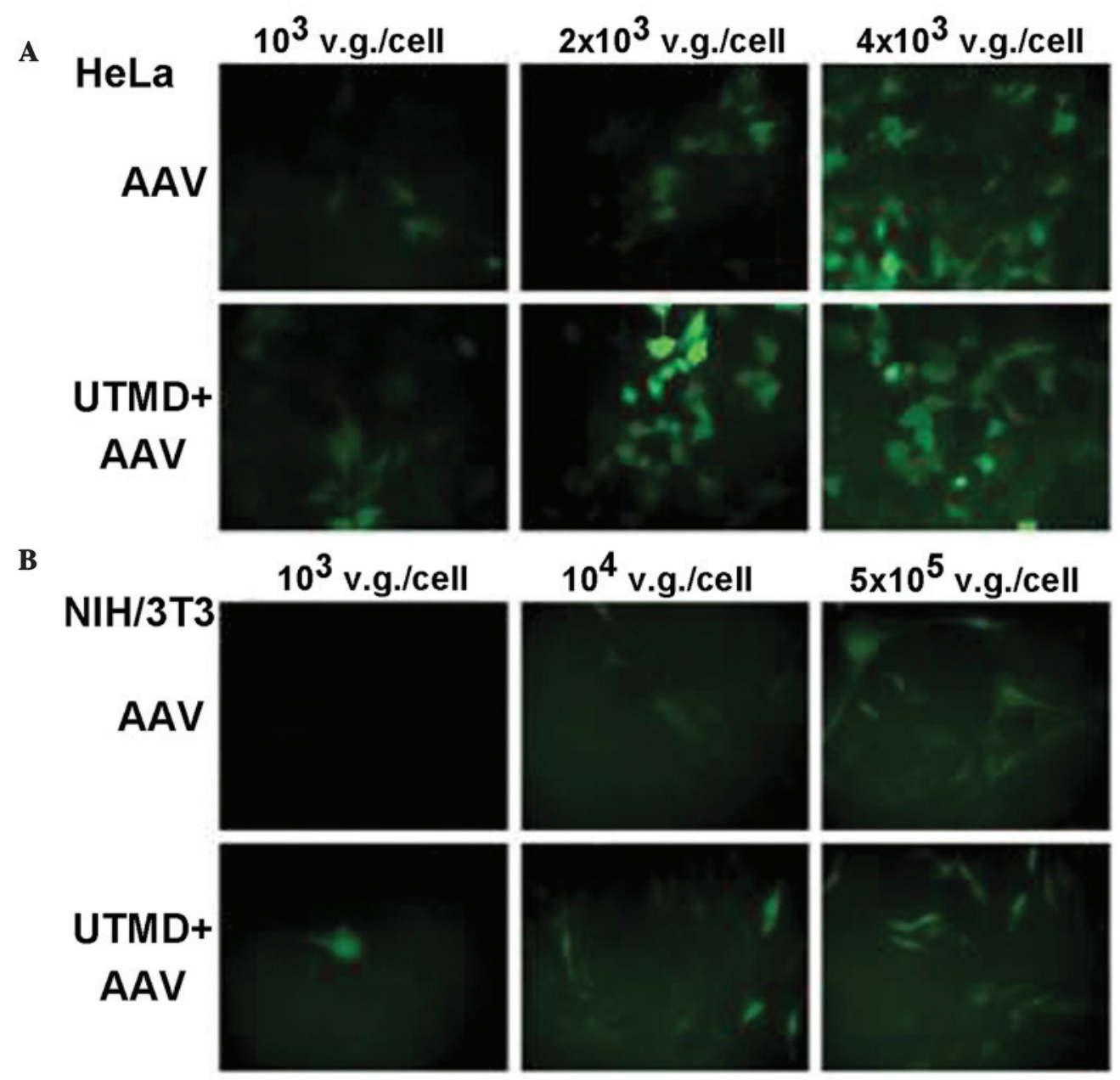

Figure 1. Fluorescence microscopy of EGFP. (A) Fluorescence microscopy of HeLa cells $48 \mathrm{~h}$ following infection with rAAV2-EGFP with three different MOIs. The cells in the AAV group were infected with rAAV2-EGFP alone. The cells in the UTMD+AAV group were infected with rAAV2-EGFP and treated with optimized UTMD. (B) Fluorescence microscopy of NIH/3T3 cells $48 \mathrm{~h}$ following infection with rAAV2-EGFP with three different MOIs. v.g., vector genome; EGFP, enhanced green fluorescent protein; rAAV2, recombinant adeno-associated virus serotype 2; MOI, multiplicity of infection; UTMD, ultrasound-targeted microbubble destruction.

to the livers of rats with hepatic fibrosis using a US-targeted cationic liposome-bearing microbubble destruction gene delivery system. The results showed that this method effectively transported the plasmids to the rat liver. The artificial miRNA was shown to reduce the pathological changes associated with hepatic fibrosis as well as the protein and mRNA expression of CTGF and transforming growth factor- $\beta 1$.

There are a number of mechanisms by which to increase cell membrane permeability that are in current use. These include using micro-circumflex and micro-fluid to punch transient holes in the surfaces of cell membranes (23-25), increasing the level of oxyradicals in cells (26) and promoting endocytosis (27) and altering the liquidity of the membrane phospholipid bilayer using US or UTMD (28). However, these mechanisms remain to be fully elucidated. Jin et al (29) suggested a further mechanism, hypothesizing that the initial stimulation of cellular endocytosis by UTMD may be mediated by grid proteins, due to the consistent peaks of endocytosis with UTMD, and those of clathrin-dependent endocytosis.

Du et al also conducted trials examining the safety of UTMD. This group attempted to identify the optimal US or UTMD settings for use in different types of tumor cells and those normal cells and tissues may be well incubated under the action of such optimal conditions (Fig. 1, taken from reference 29) (15,30-36). Zhang et al (37) demonstrated that the use of the noninvasive UTMD technique improved the localization of MSCs to the kidneys and promoted renal repair in rats with diabetic nepropathy. Other studies have been conducted in human participants into the safety of UTMD. For example, Li et al (38) found that UTMD specifically and reversibly enhanced interstitial permeability whilst having no effect on the glomerulus.

\section{Diagnosis and therapeutic application of UCA nanobubbles (NBs)}

Due to the rapid development in the field of nanotechnology, a number of NB contrast agents have been produced using liposomes, inorganic materials, metals and polymers (39). During the synthesis of NBs, the organic solvent is removed by evaporation or extraction, and the internal water-phase is eliminated during lyophilization or spray drying. NBs may possess a porous internal structure with multiple voids that are amenable to analysis with scanning or transmission electron microscopy. When they are collectively deposited 
on the surfaces of tissues or cells in a layering effect, these particles create a local acoustic impedance mismatch that produces a strong US signal without a concomitant increase in the background level. Unlike MB formulations that are naturally echogenic, these NBs do not have good inherent acoustic reflectivity $(40,41)$. Furthermore, polymer-shelled nano-sized MBs have less elastic shells, which may generate less non-linear harmonic waves. Sciallero et al (42) found that for polymeric nano-contrast agent, the optimum contrast-to-tissue ratio was obtained at an excitation pressure amplitude of $230 \mathrm{kPa}$. Although this pressure amplitude is higher than that which is conventionally used for lipid MBs, it does not cause the rupture of the polymeric nanobubble contrast agent.

Targeted imaging with NBs. Due to their small size, NB-attached ligands are important in targeted-molecular imaging (43-45). Lin et al (46) demonstrated a simple ultrasonic approach with which to produce protein-caged nanomaterials coated with MBs for use as bimodal contrast agents. Two types of protein-caged nanomaterials may be rapidly transformed into MBs with the use of high-intensity US-induced emulsification and cross-linking of protein-caged nanomaterial with bovine serum albumin in aqueous solutions. These are prepared either by a self-assembled protein corona around polymer-coated nanoparticles (referred to here as protein-caged NPs) or by protein-caged fluorescent gold nanoclusters (referred to here as protein-caged NCs). The sonochemical route produces MBs with a mean diameter of 1-3 $\mu \mathrm{m}$, which are able to safely pass through the microvasculature without diffusing across the endothelium. This transformation of versatile nanomaterials into $\mathrm{MBs}$, which comprise a gas core surrounded by a biocompatible protein/NP shell, forms nanomaterials with the added ability to scatter sound waves, offering further potential in clinical application as UCAs.

Photoacoustic molecular imaging. Recently, the use of photoacoustic molecular imaging has developed rapidly (47-50). Kim et al (51) have developed novel dual-modal contrast agent encapsulated-ink poly actic-co-glycolic acid (PLGA) MBs and NBs for photoacoustic and US imaging. Soft gelatin phantoms with embedded tumor simulators of encapsulated-ink PLGA MBs and NBs in various concentrations are visible in photoacoustic (PA) and US images. By treating the surface of the bubbles in order to target specific molecules and using in combination with PA and US imaging, these contrast agents can be used to investigate intracranial tumor boundary mapping as well as for molecular imaging of primary and metastatic tumors (52). In addition, pulsed magneto-motive US imaging (pMMUS) has been developed as a contrast-agent-assisted US-based imaging modality that is able to capture biological events at the cellular and molecular levels. During pMMUS imaging, a high intensity pulsed magnetic field is used to excite cells or tissue, which have been labeled with magnetic nanoparticles. US imaging is then used to monitor the mechanical response of the tissue to an externally applied magnetic field (53). The use of gold nanostructures in PA imaging and photothermal therapy has been extensively investigated. However, the structure of nonspherical gold nanoparticles is easily damaged following laser irradiation, and may thus lose a degree of efficacy in this context. A novel class of exogenous PA contrast agents, palladium nanosheets (PNSs) with strong optical absorption in the near-infrared (NIR) region has also been applied (54).

Multimodal imaging. Multimodal imaging has become increasingly important in the diagnosis and prognosis of a number of diseases. Currently, certain noninvasive, quantitative and functional imaging techniques are used in standard clinical practice. These include, US imaging, magnetic resonance imaging (MRI) and optical imaging (fluorescence imaging). Each of these modalities has particular advantages and disadvantages. US is a real-time, low-cost, non-ionizing and widely available imaging tool, but its resolution is low compared with other techniques and it is highly operator-dependent. MRI is useful for imaging soft tissue. It has a high spatial resolution and possesses multi-planar imaging capacities. However, the cost remains relatively high and it takes longer to obtain images than US, with relatively low sensitivity. Fluorescence imaging has high sensitivity and produces multicolor images. However, it is nonquantitative and does not penetrate tissues well. These different modalities can be mediated by nanoparticles to produce complementary methods of imaging (55). Barnett et al (56) demonstrated that perfluorocarbon nanoparticles, including rhodamine perfluorooctylbromide (PFOB) nanoparticles and rhodamine perfluoropolyether nanoparticles are multimodal cellular contrast agents, which may be amenable to development for use in real-time targeted delivery and imaging of transplanted human pancreatic islets or other cells, using MRI, US or computed tomography (CT) imaging. Furthermore, Anayama et al (57) used a nano-sized liposome-based contrast agent in ultrasonic bronchoscopies, and $\mathrm{CT}$ and fluorescence optical imaging to delineate features of a rabbit lung VX2 tumor. Contrast agents have been added to NBs so that they can be visualized using 1H MRI, 19FMRI, $\mathrm{XR} / \mathrm{CT}$ and US imaging. This has led to a novel generation of imaging biomaterials that render cells visible with multiple imaging modalities (58). Similarly, Cheng et al (59) investigated a multimodal imaging contrast agent in rat and mouse models, demonstrating via MTT and hemolysis studies that the nanodroplets used were biocompatible and safe, and exhibited significant ultrasound-triggered phase transition properties under clinical diagnostic ultrasound irradiation.

Rapoport et al (60) demonstrated that at physiological temperatures, nanodroplets converted into nanobubbles/MBs. Doxorubicin (Dox) was localized in the MB walls, which were formed by the block copolymer. Dox-loaded micelles and NBs extravasated selectively into the tumor interstitium when injected into mice. Here, the NBs coalesced to produce MBs with a strong, durable US contrast. Dox was shown to be strongly retained in the MBs but was released in response to therapeutic US. When direct US was applied, the MBs cavitated. This process significantly enhanced intracellular Dox uptake by tumor cells in vitro compared with that observed in unsonicated MBs and unsonicated micelles, and resulted in tumor regression in the mouse model.

In 2011 Ke et al (61) synthesized a new drug-loaded gold nano-microcapsule, which may be used in diagnostic imaging as well as photothermal therapy, triggered by UTMD. In 2013, Ke et al (62) also demonstrated that the multifunctional 

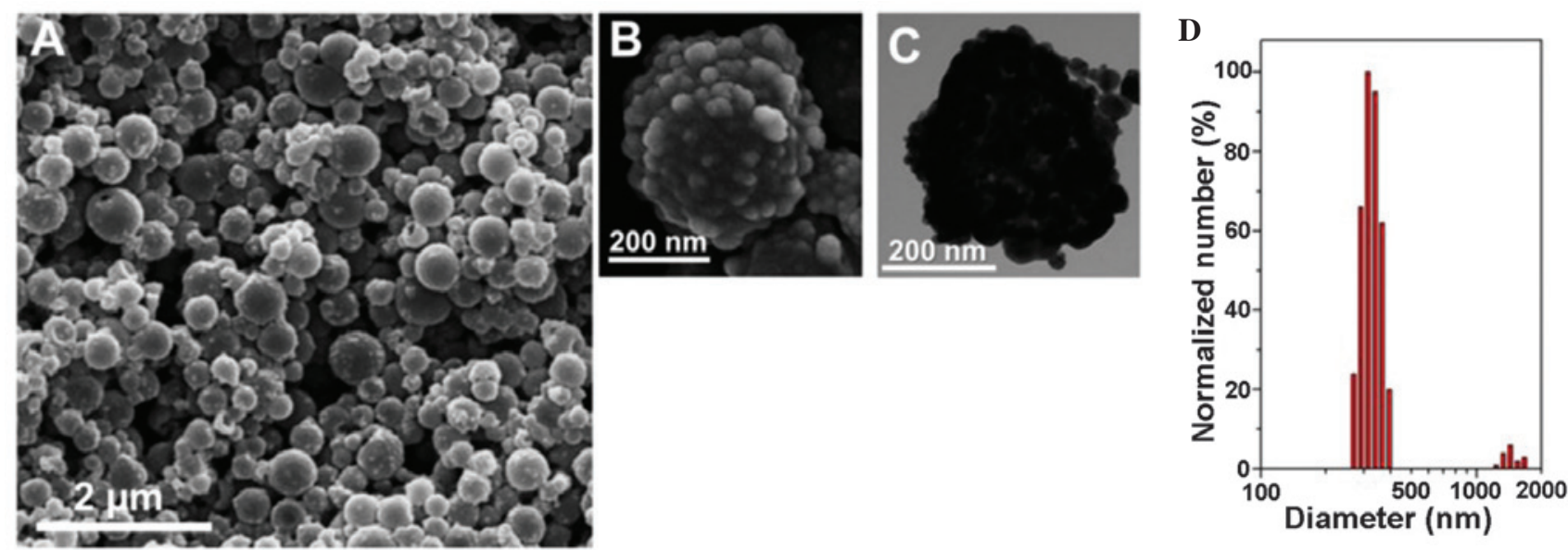

Figure 2. (A and B) Scanning electron microscopy images of the PEGylated gold nanoshelled nanocapsules; (C) transmission electron microscopy images of the agent; and (D) size distribution of the theranostic agent.

A
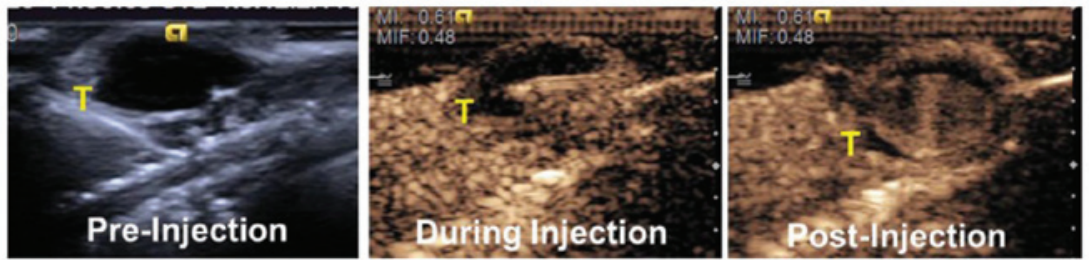

B
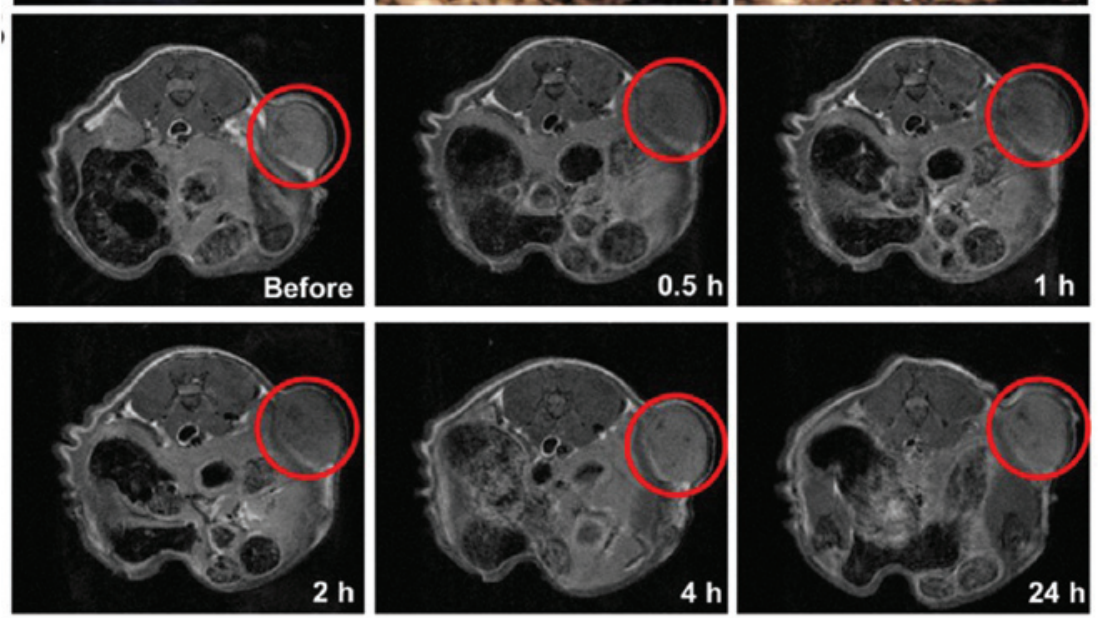

C

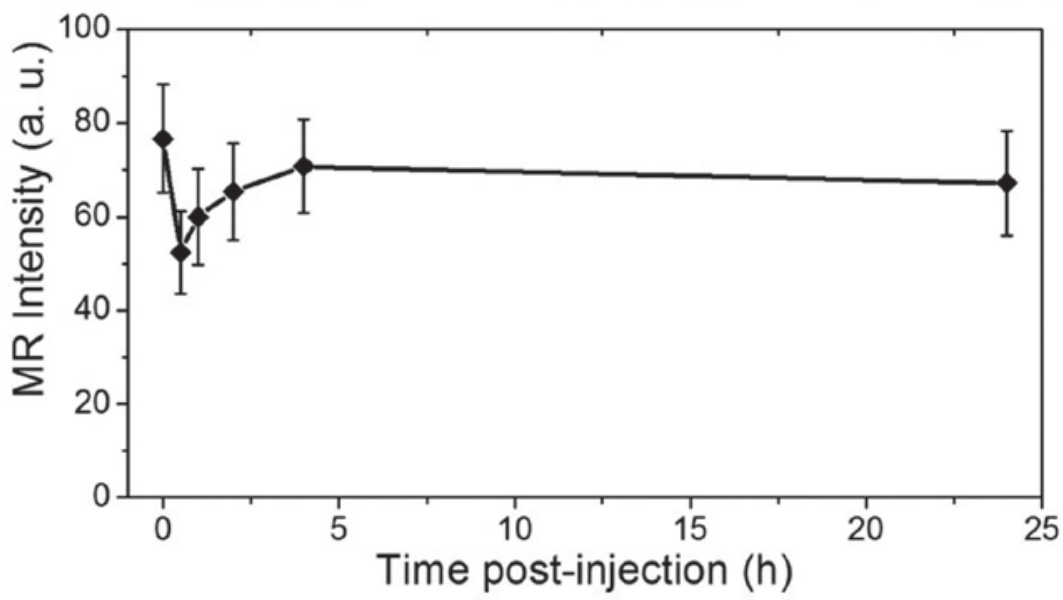

Figure 3. (A) Contrast-enhanced ultrasonograms before, during and after the intratumoral injection of the agent $(0.2 \mathrm{ml}, 2 \mathrm{mg} / \mathrm{ml}) \mathrm{into} \mathrm{mice}$ for visualization of the agent distribution to guide the subsequent therapy (tumors indicated by T). (B) T2-weighted MR images of the tumors at $0,0.5,1,2,4$ and $24 \mathrm{~h}$ following intravenous injection of the agent $(0.15 \mathrm{ml}, 2 \mathrm{mg} / \mathrm{ml})$ for visualization of tumor areas to guide the subsequent photothermal ablation (tumors are indicated by red circles). (C) MR intensity profile of the tumors from tumor-bearing mice at $0,5,10,15,20$ and $25 \mathrm{~h}$ following intravenous administration of the nanocapsules. MR, magnetic resonance. 
nanocapsules were synthesized through loading PFOB and superparamagnetic iron oxide nanoparticles into polylactic acid nanocapsules (NCs), followed by the formation of PEGylated gold nanoshell on the surface. The resulting multi-component NCs were shown to be able to act as nanotheranostic agents. These were used to achieve successful noninvasive bimodal US/MRI guided photothermal ablation in human tumor xenograft models. Such a single theranostic agent used in combination with real-time US and high-resolution MR imaging would be of great value in providing comprehensive diagnostic information and identifying the dynamics of disease progression for the accurate and timely application of localized therapy. These molecules show great potential as an effective nanoplatform for contrast imaging guided photothermal therapy (Figs. 2 and 3, taken from reference 61).

\section{Conclusion}

Multifunctional US MB/NB formulations have been rapidly developed for use in combining ultrasonic contrast enhanced imaging and passive-active targeted therapy. Their application has shown promise in the diagnosis and treatment of a variety of diseases, in particular, malignancy.

\section{Acknowledgements}

This review was supported by the National Natural Science Foundation of China (grant nos. 81171352 and 81271596).

\section{References}

1. Xing ZW, Wang J, Ke H, et al: The fabrication of novel nanobubble ultrasound contrast agent for potential tumor imaging. Nanotechnology 21: 145607, 2010.

2. Tran TD, Caruthers SD, Hughes M et al: Clinical applications of perfluorocarbon nanoparticles for molecular imaging and targeted therapeutics. Int J Nanomedicine 2: 515-526, 2007.

3. Quaia E: Microbubble ultrasound contrast agents: an update. Eur Radiol 17: 1995-2008, 2007.

4. Sun C, Sboros V, Butler MB and Moran CM: In vitro acoustic characterization of three phospholipid ultrasound contrast agents from 12 to $43 \mathrm{MHz}$. Ultrasound Med Biol 40: 541-550, 2014.

5. Pfister K, Kasprzak PM, Apfelbeck H, et al: The significance of contrast-enhanced ultrasound in vascular surgery. Zentralbl Chi: Dec 10, 2013 (Epub ahead of print) (In German).

6. Liu H, Jiang Y, Dai Q, et al: Peripheral enhancement of breast cancers on contrast-enhanced ultrasound: correlation with microvessel density and vascular endothelial growth factor expression. Ultrasound Med Biol 40: 293-299, 2014.

7. Malinova M: Preoperative sonovue contrast color Doppler in patients with cervical cancer. Preliminary report. Akush Ginekol (Sofiia) 52 (Suppl 1): 11-16, 2013 (In Bulgarian).

8. Hoffmann R, von Bardeleben S, Barletta G, et al: Comparison of two- and three-dimensional unenhanced and contrast-enhanced echocardiographies versus cineventriculography versus cardiac magnetic resonance for determination of left ventricular function. Am J Cardiol 113: 395-401, 2014.

9. Sartori S, Postorivo S, Vece FD, et al. Contrast-enhanced ultrasonography in peripheral lung consolidations: What's its actual role? World J Radiol 5: 372-380, 2013.

10. Saracco A, Szabó BK, Aspelin P, et al: Contrast-enhanced ultrasound using real-time contrast harmonic imaging in invasive breast cancer: comparison of enhancement dynamics with three different doses of contrast agent. Acta Radiol Jan 20, 2014 (Epub ahead of print).

11. Lisowska A, Knapp M, Tycinska A, et al. Usefulness of automatic measurement of contrast flow intensity: an innovative tool in contrast-enhanced ultrasound imaging of atherosclerotic carotid plaque neovascularization. A pilot study. Int Angiol 33: 50-57, 2014.
12. Nagesha D, Laevsky GS, Lampton $P$, et al: In vitro imaging of embryonic stem cells using multiphoton luminescence of gold nanoparticles. Int J Nanomedicine 2: 813-819, 2007.

13. Threlfall G, Wu HJ, Li K, et al: Quantitative guidelines for the prediction of ultrasound contrast agent destruction during injection. Ultrasound Med Biol 39: 1838-1847, 2013.

14. Chen ZY, Liang K, Lin Y and Yang F: Study of the UTMD-based delivery system to induce cervical cancer cell apoptosis and inhibit proliferation with shRNA targeting Survivin. Int J Mol Sci 14: 1763-1777, 2013.

15. Li F, Jin L, Wang H, et al: The dual effect of ultrasound-targeted microbubble destruction in mediating recombinant adeno-associated virus delivery in renal cell carcinoma: transfection enhancement and tumor inhibition. J Gene Med 16: 28-39, 2014.

16. Liu H, Chang S, Sun J, et al: Ultrasound-mediated destruction of LHRHa-targeted and paclitaxel-loaded lipid microbubbles induces proliferation inhibition and apoptosis in ovarian cancer cells. Mol Pharm 11: 40-48, 2014.

17. $\mathrm{Pu} \mathrm{C}$, Chang S, Sun J, et al: Ultrasound-mediated destruction of LHRHa-targeted and paclitaxel-loaded lipid microbubbles for the treatment of intraperitoneal ovarian cancer xenografts. Mol Pharm 11: 49-58, 2014.

18. Yan P,Chen KJ, Wu J, et al: The use of MMP2 antibody-conjugated cationic microbubble to target the ischemic myocardium, enhance Timp3 gene transfection and improve cardiac function. Biomaterials 35: 1063-1073, 2014.

19. Deng Q, Chen JL, Zhou Q, et al: Ultrasound microbubbles combined with the NFKB binding motif increase transfection efficiency by enhancing the cytoplasmic and nuclear import of plasmid DNA. Mol Med Rep 8: 1439-1445, 2013.

20. Ling ZY, Shu SY, Zhong SG, et al: Ultrasound targeted microbubble destruction promotes angiogenesis and heart function by inducing myocardial microenvironment change. Ultrasound Med Biol 39: 2001-2010, 2013.

21. Hao YN, Luo WL, Wang D and Wang ZG: Experimental research on treatment of injured facial nerves induced by hepatocyte growth factor mediated by ultrasound-targeted microbubble destruction. J Craniofac Surg 24: 421-424, 2013.

22. Yang D, Gao YH, Tan KB, et al: Inhibition of hepatic fibrosis with artificial microRNA using ultrasound and cationic liposome-bearing microbubbles. Gene Ther 20: 1140-1148, 2013.

23. Prentice P, Cushierp A, Dholakiak, et al: Membrane disruption by optically controlled microbubble cavitiation. Nat Phys 1: 107-110, 2005.

24. Tachibana K, Uchida $\mathrm{T}$, Ogawa $\mathrm{K}$, et al: Induction of cell-membrane porosity by ultrasound. Lancet 353: 1409, 1999.

25. van Wamel A, Kooiman K, Harteveld M, et al: Vibrating microbubbles poking individual cells: drug transfer into cells via sonoporation. J Control Release 112: 149-155, 2006.

26. Juffermans LJ, Dijkmans PA, Musters RJ, et al: Transient permeabilization of cell membranes by ultrasound-exposed microbubbles is related to formation of hydrogen peroxide. Am J Physiol Heart Circ Physiol 291: H1595-H1601, 2006.

27. Miller DL and Gies RA: The interaction of ultrasonic heating and cavitation in vascular bioeffects on mouse intestine. Ultrasound Med Biol 24: 123-128, 1998.

28. Schlicher RK, Radhakrishna H, Tolentino TP, et al: Mechanism of intracellular delivery by acoustic cavitation. Ultrasound Med Biol 32: 915-924, 2006.

29. Jin LF, Li F, Wang HP, et al: Ultrasound targeted microbubble destruction stimulates cellular endocytosis in facilitating adeno-associated virus delivery. Int J Mol Sci 14: 9737-9750, 2013.

30. Du J, Shi QS, Sun Y, et al: Enhanced delivery of monomethoxypoly(ethylene glycol)-poly(lactic-co-glycolic acid)-poly l-lysine nanoparticles loading platelet-derived growth factor BB small interfering RNA by ultrasound and/or microbubbles to rat retinal pigment epithelium cells. J Gene Med 13: 312-323, 2011.

31. Du J, Sun Y, Shi QS, et al: Biodegradable nanoparticles of mPEG-PLGA-PLL triblock copolymers as novel non-viral vectors for improving siRNA delivery and gene silencing. Int $\mathrm{J}$ Mol Sci 13: 516-533, 2012.

32. Shi Q, Liu P, Sun Y, et al: siRNA delivery mediated by copolymer nanoparticles, phospholipid stabilized sulphur hexafluoride microbubbles and ultrasound. J Biomed Nanotechnol 10: 436-444, 2014.

33. Jin L, Li F, Wang H, et al: Ultrasound-targeted microbubble destruction enhances gene transduction of adeno-associated virus in a less-permissive cell type, NIH/3T3. Mol Med Rep 8: 320-326, 2013. 
34. Li HL, Zheng XZ, Wang HP, et al: Ultrasound-targeted microbubble destruction enhances AAV-mediated gene transfection in human RPE cells in vitro and rat retina in vivo. Gene Ther 16: 1146-1153, 2009

35. Zheng X, Du L, Wang H and Gu Q: A novel approach to attenuate proliferative vitreoretinopathy using ultrasound-targeted microbubble destruction and recombinant adenoassociated virus-mediated RNA interference targeting transforming growth factor-b2 and platelet-derived growth factor-B. J Gene Med 14 339-347, 2012

36. Li YH, Jin LF, Du LF, et al: Enhancing HSP70-ShRNA transfection in 22RV1 prostate cancer cells by combination of sonoporation, liposomes and HTERT/CMV chimeric promoter. Int J Oncol 43: 151-158, 2013.

37. Zhang Y, Ye C, Wang G, et al: Kidney-targeted transplantation of mesenchymal stem cells by ultrasound targeted microbubble destruction promotes kidney repair in diabetic nephropathy rats. BioMed Res Int 2013: 526367, 2013

38. Li P, Gao Y, Zhang J, et al. Renal interstitial permeability changes induced by microbubble enhanced diagnostic ultrasound. J Drug Target 21: 507-514, 2013.

39. Jafari S, Diou O, Mamou J, Renault G, et al: High-frequency (20 to $40 \mathrm{MHz}$ ) acoustic response of liquid-filled nanocapsules. IEEE Trans Ultrason Ferroelectr Freq Control 61: 5-15, 2014

40. Hughes GA: Nanostructure-mediated drug delivery. Nanomedicine 1: 22-30, 2005.

41. Lanza GM, Trousil RL, Wallace KD, et al: In vitro characterization of a novel, tissue-targeted ultrasonic contrast system with acoustic microscopy. J Acoust Soc Am 104: 3665-3672, 1998.

42. Sciallero C, Paradossi G and Trucco A: A preliminary in vitro assessment of polymer-shelled microbubbles in contrast-enhanced ultrasound imaging. Ultrasonics 52: 456-464, 2012.

43. Nie L, Chen M, Sun X, et al: Palladium nanosheets as highly stable and effective contrast agents for in vivo photoacoustic molecular imaging. Nanoscale 6: 1271-1276, 2014.

44. Trung TD Tran, Caruthers SD, Hughes M, et al: Clinical applications of perfluorocarbon nanoparticles for molecular imaging and targeted therapeutics. Int J Nanomedicine 2: 515-526, 2007.

45. Milgroom A, Intrator M, Madhavan K, et al: Mesoporous silica nanoparticles as a breast-cancer targeting ultrasound contrast agent. Colloids Surf B Biointerfaces 11: 652-657, 2014.

46. Lin CA, Chuang WK, Huang ZY, et al: Rapid transformation of protein-caged nanomaterials into microbubbles as bimodal imaging agents. ACS Nano 6: 5111-5121, 2012.

47. Wilson KE, Wang TY and Willmann JK: Acoustic and photoacoustic molecular imaging of cancer. J Nucl Med 54: 1851-1854, 2013.

48. Homan KA, Souza M, Truby R, et al: Silver nanoplate contrast agents for in vivo molecular photoacoustic imaging. ACS Nano 6 : 641-650, 2012
49. Ku G, Zhou M, Song S, et al: Copper sulfide nanoparticles as a new class of photoacoustic contrast agent for deep tissue imaging at $1064 \mathrm{~nm}$. ACS Nano 6: 7489-7496, 2012.

50. Sciallero C and Trucco A: Ultrasound assessment of polymer-shelled magnetic microbubbles used as dual contrast agents. J Acoust Soc Am 133: EL478-EL484, 2013.

51. Kim C, Qin R, Xu JS, Wang LV and Xu R: Microbubbles and nanobubbles for photoacoustic and ultrasound imaging. J Biomed Opt 15: 010510, 2010.

52. Xu JS, Huang J, Qin R, et al: Synthesizing and binding dual-mode poly (lactic-co-glycolicacid) (PLGA) nanobubbles for cancer targeting and imaging. Biomaterials 31: 1716-1722, 2010.

53. Mehrmohammadi M, Shin TH, Qu M, et al: In vivo pulsed magneto-motive ultrasound imaging using high-performance magnetoactive contrast nanoagents. Nanoscale 5: 11179-11186, 2013.

54. Nie L, Chen M, Sun X, et al: Palladium nanosheets as highly stable and effective contrast agents for in vivo photoacoustic molecular imaging. Nanoscale 6: 1271-1276, 2014.

55. Park J, Park D, Shin U, et al: Synthesis of laboratory ultrasound contrast agents. Molecules 18: 13078-13095, 2013.

56. Barnett BP, Ruiz-Cabello J, Hota P, et al: Use of perfluorocarbon nanoparticles for non-invasive multimodal cell tracking of human pancreatic islets. Contrast Media Mol Imaging 6: 251-259, 2011.

57. Anayama T, Nakajima T, Dunne M, et al: A novel minimally invasive technique to create a rabbit VX2 lung tumor model for nano-sized image contrast and interventional studies. PLoS One 8: e67355, 2013

58. Arifin DR, Kedziorek DA, Fu Y, et al: Microencapsulated cell tracking. NMR Biomed 26: 850-859, 2013.

59. Cheng X, Li H, Chen Y, et al: Ultrasound-triggered phase transition sensitive magnetic fluorescent nanodroplets as a multimodal imaging contrast agent in rat and mouse model. PLoS One 8: e85003, 2013.

60. Rapoport N, Gao Z and Kennedy A: Multifunctional nanoparticles for combining ultrasonic tumor imaging and targeted chemotherapy. J Natl Cancer Inst 99: 1095-1106, 2007.

61. Ke H, Wang J, Dai Z, Jin Y, Qu E, Xing Z, Guo C, Yue X and Liu J: Gold-nanoshelled microcapsules: A theranostic agent for ultrasound contrast imaging and photothermal therapy. Angew Chem Int Ed Engl 50: 3017-3021, 2011.

62. Ke H, Wang J, Tong S, et al: Gold nanoshelled liquid perfluorocarbon magnetic nanocapsules: a nanotheranostic platform for bimodal ultrasound/magnetic resonance imaging guided photothermal tumor ablation. Theranostics 4: 12-23, 2013. 